\title{
The influence of tethering and gravity on the stability of compliant liquid-lined
} airways.

\author{
Jeremy Whang, Chandler Faulman, Thomas A. Itin and Donald P. Gaver III \\ Department of Biomedical Engineering \\ Tulane University
}

New Orleans, LA 70130

For consideration by the Journal of Biomechanics as an Original Article.

Word Count: 3491

Corresponding Author:

Donald P. Gaver

Department of Biomedical Engineering

Boggs 500

Tulane University

New Orleans, LA 70118

dpg@tulane.edu

(504) 865-5150

(C) 2016. This manuscript version is made available under the Elsevier user license 


\section{Abstract}

This study revolves around two simple questions: 1) how does pulmonary airway recruitment/de-recruitment (RecDer) depend on the tethering support provided by surrounding airways and alveoli, and 2) does airway angle of inclination ( ) influence airway stability? These two questions are critical to understanding the existence and prevention of atelectrauma, which may contribute to ventilator-induced lung injury (VILI). To address these questions, we develop PDMS 2mmID compliant tubes that mimic pulmonary airways. Airway obstruction is modeled using silicone oil, and recruitment occurs through insufflation with a constant flow of air at $Q=0.25 \mathrm{ml} / \mathrm{s}$. Parenchymal tethering is modeled through the use of a pressure chamber through which we independently establish the external pressure $\left(P_{\text {ext }}\right)$. Repetitive RecDer oscillation is observed as a function of $P_{\text {ext }}$ and. We find that airway collapse significantly increases the rate of instability, and this rate correlates strongly with the dimensionless film thickness $(=h / R)$, where $h$ is the film thickness and $R$ is the transumural pressure dependent vessel radius. Furthermore, the angle of orientation influences RecDer oscillation, with stability decreased when airflow is directed in the upward direction. These results may provide insight into protective mechanical ventilation processes that can reduce the existence or severity of VILI. 


\section{Introduction}

Respiratory distress syndrome (RDS), acute respiratory distress syndrome (ARDS), asthma and cystic fibrosis are examples of lung diseases where lining fluid abnormalities play a key role in disease etiology. For example, ARDS afflicts nearly 200,000 individuals annually in the US alone, with a mortality rate of $40 \%$. ARDS is not a 'life-style' condition - instead it arises from insults such as bacterial infection (sepsis), liquid aspiration or noxious gas inhalation. A hallmark of ARDS is the existence of fluid filled lungs with high surface tension interfaces. ARDS afflicts individuals in the prime of their life, and survivors may suffer from long-term neurological deficits, depression and decreased quality of life (Hopkins et al., 2005). Furthermore, the ICU length of stay is significantly correlated with decreased quality of life, suggesting that a reduction of ARDS severity through improved treatment can have a long-term impact on patients and their families (Hopkins et al., 2004).

Mechanical ventilation may cause ventilator-induced lung injury (VILI) because it can introduce large mechanical stresses on sensitive pulmonary tissues. The damaging effects and the potential for improvement is demonstrated by the benefits associated with reducing ventilation tidal volumes from the prior standard of $12 \mathrm{ml} / \mathrm{kg}$ to $6 \mathrm{ml} / \mathrm{kg}$ (2004). This change in ventilation standards significantly reduced mortality of ARDS from $75 \%$ to $40 \%$, and is attributed to the reduction of lung over-distension (volutrauma). VILI damage can also be caused by atelectrauma that arises due to the repeated recruitment and derecruitment (RecDer) of airways and alveoli, resulting in epithelial cell damage due to mechanical stresses associated with interfacial flows and fluid-structure interactions (Bilek 
et al., 2003; Glindmeyer et al., 2012; Huh et al., 2007; Jacob and Gaver, 2012; Kay et al., 2004).

Aligning mechanical ventilation processes with the physical capacity of the lung could impart benefits to patient survival. Accurate patient-centered alignment may require computational models that couple multi-phase flows with multi-scale models of the lung (M. Howatson Tawhai, 2000; Mullally et al., 2009; Ryans et al., 2016; Suki et al., 1994; Tawhai and Burrowes, 2008). To be tractable, such models will need to rely on robust reduced-dimension rules that can be used to provide estimates of micro-scale phenomena that interact with macro-scale behavior.

In the present study, we are focused on elucidating events associated with (de)recruitment of compliant tubes that model compliant pulmonary airways. We are specifically interested in how the transmural pressure, defined as the difference between the internal and external pressure $\left(P_{t m}=P_{\text {Int }}-P_{\text {ext }}\right)$, affects existence of repeated recruitment/de-recruitment events (RecDer oscillation) due to fluid-structure instabilities. We also investigate whether the angle of inclination affects RecDer events.

We have developed model compliant tubes that are intended to mimic the properties of pulmonary airways. We evaluate the compliance to establish the 'tube-law,' and then investigate the stability to fluid-structure instabilities within a pressure chamber to independently set $P_{E x t .}$ Below we describe the basic RecDer dynamics that we investigate. This process is described in Figure 1 and explained in detail below. 
Airway Compliance

Pulmonary airways are liquid-lined compliant vessels that convey air to and from the alveoli for gas-exchange with the environment. A general description of the compliance is provided by the 'tube law' that represents the relationship between the dimensionless cross-sectional area $\left(A / A_{0}\right)$ and the transmural pressure $\left(P_{t m}\right)$, where $A_{0}$ represents the area of the fully inflated airway. Lambert et al. (Lambert et al., 1982) mathematically described the sigmoidal behavior for central and peripheral airways. That analysis quantified the variable compliance behavior in the lung, with peripheral airways being highly compliant $\left(\frac{1}{A} \frac{d A}{d P_{t m}}\right)$ especially near $P_{t m}=0$. Figure 2 provides representative tubelaws that qualitatively describe pulmonary airway compliance based upon the analysis of Lambert et al.; this illustrates the highly compliant nature of peripheral airways in comparison to central airways. We note that any given airway has a strong $P_{t m}$ dependence to the compliance -- pulmonary airways are much less compliant when fully inflated $\left(P_{t m}>17 \mathrm{cmH}_{2} \mathrm{O}\right)$ or when fully collapsed $\left(P_{t m}<5 \mathrm{cmH}_{2} \mathrm{O}\right)$. Collapsed buckle radially, with the primary mode of instability creating a dumbbell form in untethered tubes; however other modes may dominate if tethering exists (Flaherty, 1972). In the present study we will develop in vitro models of untethered compliant airways that can be used to investigate these collapse mechanisms.

\section{Recruitment}

Airway recruitment occurs when a fluid obstructed airway is reopened by the penetration of a finger of air that displaces the liquid and obstruction (Figure 1). Many experimental and theoretical investigations have explored the behavior of recruitment in rigid and 
flexible models, and an array of phenomena exists based upon the volume of the obstruction, the airway wall flexibility, the surface tension, the existence of surfactant, the viscosity of the fluid and the recruitment velocity (Gaver et al., 1996; Gaver III et al., 1990; Ghadiali and Gaver III, 2000; Ghadiali and Gaver III, 2003; Halpern and Gaver, 2012; Yap and Gaver III, 1998). A key dimensionless parameter that describes the flow field is the capillary number,

$$
C a=\frac{U}{},
$$

which represents the relationship between viscous and surface tension interactions. Here is the viscosity, $U$ is the reopening viscosity and is the surface tension. The volume of fluid retained in the vessel is of critical importance to RecDer phenomena. In a rigid tube, the foundational work of Taylor (Taylor, 1961) empirically established the dimensionless film thickness that is accurate over the range $10^{4}<C a<10^{1}$ :

$$
=\frac{h}{R}=0.5 C a^{1 / 2},
$$

where $h$, is the residual film thickness and $R$ is the tube radius. This behavior is modified by the nature of closure, but it provides a framework by which one can develop a basic understanding of recruitment/de-recruitment stability.

Plateau-Rayleigh Instability

The Plateau-Rayleigh instability is hypothesized to be responsible for the occlusion of liquid-lined pulmonary airways (Campana and Saita, 2006; Cassidy et al., 1999; Heil et al., 2008; Kamm and Schroter, 1989). Briefly, this surface-tension-induced instability relates to 
the interfacial pressure drop on the air-liquid interface that coats the interior surface of the airway, which is a function of the local curvature. Statically, Laplace's law is

$$
P=\left(\frac{1}{R_{1}}+\frac{1}{R_{2}}\right)
$$

where $P$ is the interfacial pressure drop, and $R_{1}$ and $R_{2}$ are radii of curvature in orthogonal directions (for example, $R_{1} \sim$ the local radius of the interface in the radial direction and $R_{2}$ is related to the wavelength of the interfacial perturbation in the axial direction ). In gravity-free systems, theory and experiments have demonstrated that a liquid-lined vessel will obstruct due to interfacial instabilities if the dimensionless film thickness $\quad \frac{h}{R}>{ }_{\text {crit }}$, where $h$ is the local film thickness and $R$ is the radius of the tube. These studies show that $0.09<{ }_{c r i t}<0.12$, and that the vessel will obstruct by the formation of a liquid plug with a time-scale that depends upon , and (Gauglitz and Radke, 1988) and can be influenced by the existence of surfactant (Cassidy et al., 1999). Depending upon the compliance of the vessel, the system may exhibit compliant collapse, where variation of $P_{t m}$ induces a change in the local tube radius and may cause tube buckling in the region of the obstruction, as shown at negative values of $P_{t m}$ in Figure 2.

\section{Recruitment/De-recruitment Dynamics}

We explore the existence of repetitive RecDer oscillation in compliant airways (Figure 1).

To do so, we investigate the recruitment of a compliant vessel by a constant flow-rate of air, $Q$. At the initiation of the experiment a finger of air displaces a liquid obstruction that completely fills the lumen of the compliant tube. Subsequently, the remaining lining fluid and airway wall can become unstable, causing the vessel to re-obstruct (de-recruit), 
generally in a compliant collapse configuration (depending upon $P_{t m}$ ). After this initial RecDer event, the vessel may enter into a repetitive cycling of closure and reopening. In this investigation, we explore the following questions related to this phenomenon:

1) How is RecDer oscillation affected by the external pressure, $P_{E x t}$ that models the parenchymal support provided by surrounding airways and alveoli (Fujioka et al., 2013; Lai-Fook et al., 1978; Menkes et al., 1972)?

2) Does orientation with respect to gravity play a role in recruitment/de-recruitment dynamics?

\section{Materials and Methods}

Compliant Airway Development

We developed a small diameter compliant vessel of approximately $2.0 \mathrm{~mm}$ ID when fully inflated to be consistent with pulmonary airways that is transparent for visualization. We elected to construct this vessel using polydimethylsiloxane (PDMS) for which we have experience in the development of microfluidic models of aspects of the pulmonary system (Yamaguchi et al., 2014).

Compliant PDMS tubes were created with Sylgard 184 silicone elastomer base and Sylgard 184 silicone curing agent (Dow Corning Corporation; Midland, MI) mixed at a 10:1 ratio. To achieve the cylindrical geometry, a steel mandrel metal rod with an outer diameter of $2.0 \mathrm{~mm}$ was used (setting the tube ID, which equals $2 R_{0}$ ). The mandrel was first coated in diluted detergent (Colgate-Palmolive; New York, NY) to lubricate the rod. To obtain a uniform wall thickness, we constructed a device made of acrylic plates and rods with a center-plate that is used to sweep-away excess PDMS from the mandrel (Figure 3) 
After coating the mandrel in PDMS and removing air bubbles, the mandrel was placed into the device between the foundation and top plates. The central plate was lifted upwards to set the wall thickness by clearing excess PDMS. Subsequently, the mandrel with uniform PDMS was cured at $85^{\circ} \mathrm{C}$ for 45 minutes. To connect the tubes to our apparatus, we added a short segment of Tygon silicon tubing (Cole-Parmer; Vernon Hills, IL) on each end, and then adhered this to the flexible section with a small portion of PDMS. This was then cured to hold the adapter in place. Afterwards the tube was gently removed from the mandrel and flushed with $\mathrm{H}_{2} \mathrm{O}$ to remove detergent residue. This process allowed us to create flexible tubes with a wall thickness of $0.2 \mathrm{~mm}$.

We evaluated the tube compliance by filling the tube with $\mathrm{H}_{2} 0$ and connected it to a manometer, using a viscous liquid indicator droplet in the manometer tubing to evaluate the change of volume. Since the external pressure is $P_{a t m}$, the transmural pressure is simply $P_{t m}=g H$, where $H$ is the level of the fluid column in the manometer (and can be negative). In addition, $V=\left(R^{2}\right) L$, where $R$ is the inner radius of the manometer tubing, and $L$ is the change of indicator plug location.

Pressure chamber for modification of $\mathrm{P}_{\text {Ext. }}$. In order to independently set the transmural pressure and angle of orientation, we designed and constructed a pressure vessel to set $P_{\text {ext. }}$ The cylindrical chamber was constructed, as shown in Figure 4, of polyvinyl chloride (PVC) and has dimensions of 2.35 in x 12 in. A syringe is used to set $P_{E x t}$, which was monitored with a Honeywell HSCDRRN005PD2A5 differential pressure transducer (Honeywell; Morristown, NJ), and 
controlled via an Arduino microcontroller. Pressure calibration confirmed the linearity and accuracy of the transducer over the range of pressures in this study.

\section{Experimental Protocol}

Experiments were conducted with silicon oil as the occlusion fluid (Hatco 2300 synthetic lubricant, Hatco Corporation; Fords, NJ) with $\quad=20 \mathrm{~g} / \mathrm{cm} \square \mathrm{s}, \quad=33.5 \mathrm{dyn} / \mathrm{cm}$. The flowrate was adjusted to $\mathrm{Q}=0.25 \mathrm{~cm}^{3} / \mathrm{s}$ using the syringe pump, setting a nominal recruitment velocity of $U=8 \mathrm{~cm} / \mathrm{s}$ in the compliant airway under fully inflated (high $P_{t m}$ ) conditions. This initiates recruitment at $\mathrm{Ca} \sim 5 \times 10^{-2}$, which is sufficient to induce unstable recruitment (Cassidy et al., 1999). Experiments were conducted with the following independent parameters:

- Angle of Inclination: $-90^{\circ} \leq \theta \leq 90^{\circ}$ with $\Delta \theta=30$ and $\theta= \pm 15^{\circ}$

- External Pressure: $P_{E x t}=30,15,5,-15 \mathrm{cmH}_{2} \mathrm{O}$

- 4-6 tubes investigated, with $n=5$ trials per $P_{E x t}$ and

Video recordings of RecDer events were conducted for $T=1$ minute at each $\quad$ and $P_{\text {Ext. }}$. These videos were post-processed to evaluate the frequency of recruitment/derecruitment for each trial.

\section{Results}

Experiments were conducted in compliant airways with an average tube-law behavior shown in Figure 2. While these airways are not as compliant as peripheral airways, they approximate the behavior of generations 3-5, as described by Lambert et al. (Lambert et al., 1982). We evaluated the RecDer frequency for a range of $P_{e x t}$ and inclination angles, . Figure 5 demonstrates the very strong dependence of the RecDer frequency on $P_{E x t}$. For 
example, the oscillation frequency increases by approximately a factor of 5-10 from fully inflated airways $\left(P_{E x t}=-15 \mathrm{cmH}_{2} 0\right)$ to compressed airways $\left(P_{E x t}=30 \mathrm{cmH}_{2} 0\right)$. While we do not know the precise values of $P_{t m}$ because of the time-dependent internal pressures, the large values of $P_{E x t}$ are associated with $P_{t m}<0$; this leads to significantly increasing the rate of recruitment/de-recruitment due to compression of the airway that reduces the airway cross-sectional area (Figure 2).

In addition, we observe a dependence of the oscillation frequency on the angle of inclination, . Our data demonstrate that the RecDer frequency is reduced when the flow is directed in the downward direction $(<0)$. For example, at the fully inflated condition $\left(P_{E x t}=-15 \mathrm{cmH}_{2} 0\right)$ a change in orientation can result in more than a five-fold increase in oscillation frequency (albeit with small $f$ ). These trends continue for more highly compressed airways $\left(P_{E x t}=-30 \mathrm{cmH}_{2} 0\right)$, where orientation can result in a two-fold change in oscillation rate from $f=0.15 \mathrm{~Hz}$ with a downward oriented airway, to $f=0.3 \mathrm{~Hz}$ if the same airway is oriented upward with respect to the direction of flow.

Since the data at fixed $P_{\text {Ext }}$ suggest a sigmoidal representation, we fit the data to the relationship

$$
f=f_{\text {min }}+\frac{f_{\text {max }} f_{\text {min }}}{\left.1+e^{k(} 0\right)}
$$

where $\theta$ and $\theta_{0}$ are measured in degrees. 
The results are presented in Figure 5 with coefficients presented in Table 1, which show a strong correlation between the fitting function and data, with $\mathrm{R}^{2}>0.85$. We note that coefficients for the curve-fit of $P_{E x t}=15 \mathrm{cmH}_{2} 0$ data are ambiguous, with an unreasonable value of ${ }_{0}$, and hence $f_{\min }$ and $f_{\max }$ are out of range from the data. This is likely because the data deviate from the obvious sigmoidal trend - by inspection $f_{\min } \sim 0.15 \mathrm{~Hz}$ and $f_{\max } \sim$ $0.3 \mathrm{~Hz}$ at $P_{E x t}=15 \mathrm{cmH}_{2} 0$.

\section{Discussion}

To elucidate the trends observed in our experiments, we use first-order approximations to estimate the aspects that influence the stability of the system. To do so, the following assumptions are made:

1) The flow and capillary behavior is influenced by the average cross-sectional radius of the tube, $\mathrm{R}_{e f f}=\sqrt{\frac{A}{-}}$, with $A$ determined by the tube-law (Figure 2).

2) We neglect non-uniform disturbances.

3) The surface tension is constant ( = 33.5 dyn $/ \mathrm{cm})$.

4) The silicone oil has a uniform viscosity ( $=0.20 \mathrm{~g} / \mathrm{cm} \square \mathrm{s}$ ).

5) The residual film thickness, $h$, after recruitment is solely a function of $\mathrm{Ca}$ and is uniformly distributed.

The Influence of $\mathrm{P}_{\mathrm{Ext}}$

We modified $P_{E x t}$ to influence the vessel compliance, as shown in Figure 2. The compliance of the system causes the cross-sectional area to be reduced from the fully inflated state 
unless $P_{t m}$ is large (negative $P_{E x t}$ ). We found RecDer instability under all cases when $Q=0.25$ $\mathrm{ml} / \mathrm{s}$, but the oscillation frequency $f$ was highly dependent on $\mathrm{P}_{\text {Ext. }}$

The airway will become unstable to Plateau-Rayleigh instabilities if, upon recruitment, a sufficient volume of liquid remains so that a plug of fluid can develop. Gauglitz and Radke (Gauglitz and Radke, 1988) estimated that the critical dimensionless film thickness for derecruitment is $0.09<{ }_{\text {crit }}<0.12$. Cassidy et al. (Cassidy et al., 1999) used rigid tube models of airway recruitment and found experimentally that the critical capillary number for recruitment that would result in subsequent de-recruitment was $C a>1.75 \times 10^{-2}$.

We will evaluate our data to determine whether it is consistent with the hypothesis that the dimensionless film thickness correlates with the RecDer instability. A simulation of the residual film thickness after the initial reopening is beyond the scope of this study, so we consider two limiting cases:

1) The minimum dimensionless film thickness $\left({ }_{\min }\right)$ : we assume that $P_{E x t}$ sets the cross-sectional area of the tube (following the tube law), and that this is unperturbed by the recruitment event. This is equivalent to stating that $P_{I n t}=0$ when the viscous occlusion is removed (an underestimate). The dimensionless film thickness following Taylor (Taylor, 1961) is thus

$$
\min =\frac{h}{R_{e f f}\left(P_{E x t}\right)}=0.5 C a^{1 / 2}=0.5\left(\frac{Q}{R_{e f f}^{2}\left(P_{E x t}\right)}\right)^{1 / 2}
$$


2) The maximum dimensionless film thickness $\left({ }_{\max }\right)$ : we assume that the recruitment event requires a large internal pressure to displace the viscous liquid obstruction. So, $P_{\text {Int }} \gg P_{E x t}$ during the recruitment phase (an overestimate). The film is thus deposited on the fully inflated tube of radius $R_{0}$. This volume of liquid film is conserved post-recruitment $\left(P_{\text {Int }} \sim 0\right)$ and the tube collapses under the influence of $P_{\text {Ext. Using this approach, }}$

$$
\max =\left(\frac{R_{0}}{R_{e f f}\left(P_{E x t}\right)}\right)^{2 / 3} \text { min } .
$$

Using these two extreme estimates of , Figure 6 presents the oscillation period $v s$. dimensionless film thickness using the horizontal tube data $(=0)$.

The trends for both representations are similar and show that collapsing the airway (with a large $P_{E x t}$ ) significantly increases the instability (decreases the oscillation period), which correlates with an increase in the dimensionless film thickness, . So, not surprisingly, the collapse of the flexible airway increases the RecDer instability. Furthermore, if the airway is 'stented open' by a large transmural pressure $\left(P_{t m}=P_{\text {Int }}-P_{E x t}>0\right)$, the flexible airway is much less susceptible to fluid-structure instabilities, as demonstrated by a large increase in the oscillation period. Most interestingly, we observe an abrupt increase in the oscillation period at $\sim 0.1$, which is consistent with Gauglitz and Radke's estimate of ${ }_{c r i t}$. This result suggests that it will be important to maintain a positive $P_{t m}$ and low reopening velocities in order to recruit airways and reduce the likelihood of de-recruitment by sustaining a small 
. This principle is consistent with the use of positive end expiratory pressure (PEEP) ventilation. Future studies should investigate whether this trend can exist by reducing $Q$.

Influence of Angle of Inclination ( $\theta$ )

Figure 6 demonstrates that at a fixed $P_{E x t}$, an increase in the angle of inclination increases the frequency of oscillation (leading to a more unstable system). We speculate that this behavior is a result of a buoyancy effect that perturbs the internal pressure $\left(P_{I n t}\right)$ as a function of the angle of inclination. Figure 8 illustrates this effect, and suggests that the rising bubble $(>0)$ will require a reduction of $P_{\text {Int }}$ due to buoyancy. This buoyancy effect will reduce $P_{t m}$ and make the airway less stable. Likewise, when the finger of air is forced downward $(>0)$, a greater $P_{\text {Int }}$ is required to compete with buoyancy. This increases $P_{t m}$, stenting the tube in a less flaccid configuration and increasing the stability.

We hypothesize that the sigmoidal effect of should be most significant for highly collapsed tubes $\left(P_{E x t}>0\right)$ since a change in $P_{\text {int }}$ would then affect a larger change in the structure. Figure 5 demonstrates that this effect is most prominent at high compression prominent for $P_{E x t}=30 \mathrm{cmH} 20$; however, the sigmoidal behavior is observed for all values

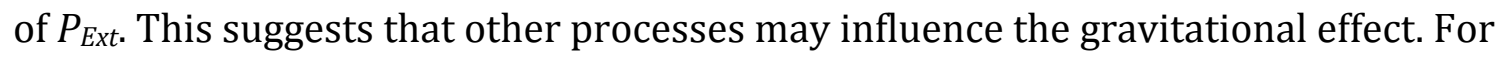
example, we speculate that the -dependence may also be a result of draining flows coupled to air-flow induced shear stress that could modulate the Plateau-Rayleigh instability similar to that investigated in (Halpern and Grotberg, 2003). 
We further observe that the buoyancy effect is more prominent for fully opened tubes ( $\mathrm{P}_{\mathrm{Ext}}$ $=-15 \mathrm{cmH} 20$ ), where the frequency varies over an eight-fold range of frequency from $0.01<\mathrm{f}<0.08 \mathrm{~Hz}$. In contrast, more fully collapsed tubes, while being unstable in general, demonstrated only a two-fold change in oscillation frequency with the range $0.15<\mathrm{f}<0.3 \mathrm{~Hz}$. To further understand the relationship of buoyancy, we calculate the Bond number

$$
B o=\underline{g D^{2}}
$$

which provides the ratio of gravitational to surface tension forces. For fully expanded tubes $\left(\mathrm{P}_{\mathrm{Ext}}=-15 \mathrm{cmH}_{2} 0\right), B o \sim 1$, so gravity and surface tension play nearly equivalent roles. In contrast, for compressed tubes $\left(\mathrm{P}_{\mathrm{Ext}}=+30 \mathrm{cmH}_{2} 0\right), B o \sim 0.05$. This implies that the effect of buoyancy is substantially decreased with collapsed vessels, a behavior that is consistent with our observations where a change in inclination has a proportionally smaller effect.

\section{Limitations}

The current study is limited for a number of reasons. First, we only investigate a single model of collapsible tube at a single $R_{0}$, and this tube this does not model the full range of compliance that exists in pulmonary airways. Second, the vessel is constructed of PDMS, which has a hydrophobic quality. Using silicone oil allowed the lining fluid to wet the tube, but may not have replicated the wetting properties of lining fluid in pulmonary airways. Third, we have correlated our results to predictions of the dimensionless film thickness, , based upon the displacement of a fully obstructed rigid tube by a finger of air, which initiates the system. This neglects the change of volume of liquid that may exist from repetitive recruitment events, which will be influenced by the motion of the liquid plug (Fujioka et al., 2008). Fourth, we did not explore the effect of dynamic surface tension, 
which would exist with reopening and closure (Halpern et al., 2008) (Gaver III et al., 2005). Follow-up studies should investigate the behavior with dynamic surface tension as influenced by surfactant under (patho)physiological conditions. Finally, the studies should investigate a variety of steady and pulsatile flow-rates. Despite these limitations, we believe that these studies provide insight into the existence of RecDer oscillation from which a full parametric investigation could be based.

\section{Conclusions}

In this study, we describe the creation of a compliant airway model that serves as a representation of a respiratory passageway. This model was used to explore the existence of repeated recruitment/de-recruitment (RecDer) events that may cause atelectrauma in the lung. This study demonstrated the strong relationship between the transmural pressure and the existence of RecDer, and suggests that ventilation management should consider the development of non-conventional ventilation protocols that retain high $P_{t m}$ so as to reduce the likelihood of atelectrauma (Emr et al., 2013). Doing so while simultaneously protecting the lung from volutrauma is challenging, especially due to regional variation of the pleural pressure. Furthermore, this study indicated that the angle of orientation could influence the stability characteristics. This result provides insight into the susceptibility of specific airways to RecDer oscillation, and may help to provide guidance towards patient postural orientation (and variation thereof) during mechanical ventilation. 


\section{Acknowledgements}

National Science Foundation Grant CBET-1033619 and Research Traineeship Grant DMS-

1043626 supported this study. Funding support for T.A.I was provided by Tulane's Center for Engaged Learning and Teaching. 


\section{References}

2004. ARDS Clinical Network: The Assesment of Low Tidal Volume and Elevated Endexpiratory Volume to Obviate Lung Injury (ALVEOLI) trial (http://www.ardsnet.org/ards04.php).

Bilek, A.M., Dee, K.C., Gaver, D.P., 3rd, 2003. Mechanisms of surface-tension-induced epithelial cell damage in a model of pulmonary airway reopening. J Appl Physiol 94, 770783.

Campana, D.M., Saita, F.A., 2006. Numerical analysis of the Rayleigh instability in capillary tubes: The influence of surfactant solubility. Phys. Fluids 18.

Cassidy, K.J., Halpern, D., Ressler, B.G., Grotberg, J.B., 1999. Surfactant effects in model airway closure experiments. J Appl Physiol 87, 415-427.

Emr, B., Gatto, L.A., Roy, S., Satalin, J., Ghosh, A., Snyder, K., Andrews, P., Habashi, N., Marx, W., Ge, L., Wang, G., Dean, D.A., Vodovotz, Y., Nieman, G., 2013. Airway pressure release ventilation prevents ventilator-induced lung injury in normal lungs. JAMA Surg 148, 10051012.

Flaherty, J.E., Keller, J.B., Rubinow, S.I., 1972. Post buckling behavior of elastic tubes and rings with opposite sides in contact. SIAM J. Appl. Math. 23, 446-455.

Fujioka, H., Halpern, D., Gaver, D.P., 3rd, 2013. A model of surfactant-induced surface tension effects on the parenchymal tethering of pulmonary airways. Journal of biomechanics 46, 319-328.

Fujioka, H., Takayama, S., Grotberg, J.B., 2008. Unsteady propagation of a liquid plug in a liquid-lined straight tube. Phys Fluids (1994) 20, 62104.

Gauglitz, P.A., Radke, C.J., 1988. An extended evolution equation for liquid-film break up in cylindrical capillaries. Chem. Eng. Sci. 43, 1457-1465.

Gaver, D.P., Halpern, D., Jensen, O.E., Grotberg, J.B., 1996. The steady motion of a semiinfinite bubble through a flexible-walled channel. J. Fluid Mech. 319, 25-65.

Gaver III, D.P., Halpern, D., Jensen, O.E., 2005. Surfactant and Airway Liquid Flows, in: Nag, K. (Ed.), Molecular Mechanisms in Lung Surfactant (Dys)function. Marcel Dekker, New York, pp. 187-223.

Gaver III, D.P., Samsel, R.W., Solway, J., 1990. Effects of surface tension and viscosity on airway reopening. J. Appl. Physiol. 69, 74-85.

Ghadiali, S.N., Gaver III, D.P., 2000. An investigation of pulmonary surfactant physicochemical behavior under airway reopening conditions. J Appl Physiol 88, 493-506. Ghadiali, S.N., Gaver III, D.P., 2003. The influence of non-equilibrium surfactant dynamics on the flow of a semi-infinite bubble in a rigid cylindrical tube. J. Fluid Mech. 478, 165-196. Glindmeyer, H.W.t., Smith, B.J., Gaver, D.P., 3rd, 2012. In situ enhancement of pulmonary surfactant function using temporary flow reversal. J. Appl. Physiol. 112, 149-158.

Halpern, D., Fujioka, H., Takayama, S., Grotberg, J.B., 2008. Liquid and surfactant delivery into pulmonary airways. Respir Physiol Neurobiol 163, 222-231.

Halpern, D., Gaver, D.P., 2012. The influence of surfactant on the propagation of a semiinfinite bubble through a liquid-filled compliant channel. J. Fluid Mech. 698, 125-159. Halpern, D., Grotberg, J.B., 2003. Nonlinear saturation of the Rayleigh instability due to oscillatory flow in a liquid-lined tube. J. Fluid Mech. 492, 251-270.

Heil, M., Hazel, A.L., Smith, J.A., 2008. The mechanics of airway closure. Resp Physiol Neurobi 163, 214-221. 
Hopkins, R.O., Weaver, L.K., Chan, K.J., Orme, J.F., 2004. Quality of life, emotional, and cognitive function following acute respiratory distress syndrome. J Int Neuropsych Soc 10, 1005-1017.

Hopkins, R.O., Weaver, L.K., Collingridge, D., Parkinson, R.B., Chan, K.J., Orme, J.F., 2005. Two-year cognitive, emotional, and quality-of-life, outcomes in acute respiratory distress syndrome. Am. J. Respir. Crit. Care Med. 171, 340-347.

Huh, D., Fujioka, H., Tung, Y.C., Futai, N., Paine, R., 3rd, Grotberg, J.B., Takayama, S., 2007. Acoustically detectable cellular-level lung injury induced by fluid mechanical stresses in microfluidic airway systems. Proc Natl Acad Sci U S A 104, 18886-18891.

Jacob, A.M., Gaver, D.P., 3rd, 2012. Atelectrauma disrupts pulmonary epithelial barrier integrity and alters the distribution of tight junction proteins ZO-1 and claudin 4. J. Appl. Physiol. 113, 1377-1387.

Kamm, R.D., Schroter, R.C., 1989. Is airway closure caused by a liquid film instability? Respir. Physiol. 75, 141-156.

Kay, S.S., Bilek, A.M., Dee, K.C., Gaver, D.P., 2004. Pressure gradient, not exposure duration, determines the extent of epithelial cell damage in a model of pulmonary airway reopening. J Appl Physiol 97, 269-276.

Lai-Fook, S.J., Hyatt, R.E., Rodarte, J.R., 1978. Effect of parenchymal shear modulus and lung volume on bronchial pressure-diameter behavior. J. Appl. Physiol. 44, 859-868.

Lambert, R.K., Wilson, T.A., Hyatt, R.E., Rodarte, J.R., 1982. A computational model for expiratory flow. J Appl Physiol 52, 44-56.

M. Howatson Tawhai, A.J.P., P. J. Hunter, 2000. Generation of an Anatomically Based ThreeDimensional Model of the Conducting Airways. Ann Biomed Eng 28, 793-802.

Menkes, H., Gamsu, G., Schroter, R., Macklem, P.T., 1972. Interdependence of lung units in isolated dog lungs. J. Appl. Physiol. 23, 675-680.

Mullally, W., Betke, M., Albert, M., Lutchen, K., 2009. Explaining Clustered Ventilation

Defects Via a Minimal Number of Airway Closure Locations. Ann. Biomed. Eng. 37, 286-300. Ryans, J., Fujioka, H., Halpern, D., Gaver, D.P., 2016. Reduced-Dimension Modeling Approach for Simulating Recruitment/De-recruitment Dynamics in the Lung. Ann. Biomed. Eng., 1-13. Suki, B., Barabasi, A.-L., Hantos, Z., Petak, F., Stanley, H.E., 1994. Avalanches and power-law behaviour in lung inflation. Nature 368, 615-618.

Tawhai, M.H., Burrowes, K.S., 2008. Multi-scale Models of the Lung Airways and Vascular System, in: Poulin, M.J., Wilson, R.J.A. (Eds.), Integration in Respiratory Control: From Genes to Systems. Springer New York, New York, NY, pp. 190-194.

Taylor, G.I., 1961. Deposition of a viscous fluid on the wall of a tube. J. Fluid Mech. 10, 161165.

Yamaguchi, E., Giannetti, M.J., Van Houten, M.J., Forouzan, O., Shevkoplyas, S.S., Gaver, D.P., 3rd, 2014. The unusual symmetric reopening effect induced by pulmonary surfactant. J Appl Physiol (1985) 116, 635-644.

Yap, D.Y.K., Gaver III, D.P., 1998. The influence of surfactant on two-phase flow in a flexiblewalled channel under bulk equilibrium conditions. Phys. Fluids 10, 1846-1863. 


\section{Table and Figure Captions}

Table 1: Coefficients to sigmoidal curve-fit of oscillation frequency following eon (4). *Note that the form for $\mathrm{P}_{\mathrm{Ext}}=15 \mathrm{cmH} 20$ is ambiguous, as discussed in the text.

Figure 1: Schematic of the Recruitment/De-recruitment (RecDer) dynamics that are investigated in this model. Note that although not illustrated, the tube is compliant and may undergo cross-sectional buckling as a result of a negative transmural pressure.

Figure 2: Representative compliance representation (tube-law) representing a change in cross-sectional area $\left(\mathrm{A} / \mathrm{A}_{0}\right)$ as a function of transmural pressure, $\mathrm{P}_{\mathrm{tm}}$. Bold lines represent pulmonary airways, and data and narrow line represents the PDMS tube used in this study.

Figure 3: Device that creates uniform thickness in the PDMS tubes. Not to scale.

Figure 4: Rendering of the entire pressure chamber. Not to scale.

Figure 5: Average interfacial instability frequency vs. for $P_{E x t}=30,15,5$ and $-15 \mathrm{cmH}_{2} 0$.

Figure 6: Relationship between the oscillation period and the estimated dimensionless film thickness.

Figure 7: Schematic of a finger of air in a compliant tube model at angles of inclination $\theta= \pm 90^{\circ}$. An increase in $P_{\text {Int }}$ is hypothesized to improve the stability and reduce RecDer oscillation. 


\begin{tabular}{|l|l|l|l|l|l|}
\hline $\begin{array}{l}P_{\text {Ext }} \\
\left(\mathrm{cm} \mathrm{H}_{2} \mathrm{O}\right)\end{array}$ & $\begin{array}{l}\mathrm{f}_{\min } \\
\left(\mathrm{s}^{-1}\right)\end{array}$ & $\begin{array}{l}\mathrm{f}_{\max } \\
\left(\mathrm{s}^{-1}\right)\end{array}$ & $\begin{array}{l}\mathrm{k} \\
\left(\text { degrees }^{-1}\right)\end{array}$ & $\begin{array}{l}\theta_{0} \\
(\text { degrees })\end{array}$ & $\mathrm{R}^{2}$ \\
\hline 30 & 0.161 & 0.316 & $4.1 \times 10^{-2}$ & 33.6 & 0.934 \\
\hline $15^{*}$ & $9.2 \times 10^{-2 *}$ & $4.40^{*}$ & $7.51 \times 10^{-3^{*}}$ & $490^{*}$ & $0.975^{*}$ \\
\hline 5 & $6.24 \times 10^{-2}$ & 0.122 & $6.73 \times 10^{-2}$ & -45.1 & 0.853 \\
\hline-15 & $8.95 \times 10^{-3}$ & 0.0745 & $5.3 \times 10^{-2}$ & -28.7 & 0.961 \\
\hline
\end{tabular}

Table 1: Coefficients to sigmoidal curve-fit of oscillation frequency following eon (4). ${ }^{*}$ Note that the form for $P_{E x t}=$ $15 \mathrm{cmH} 20$ is ambiguous, as discussed in the text. 


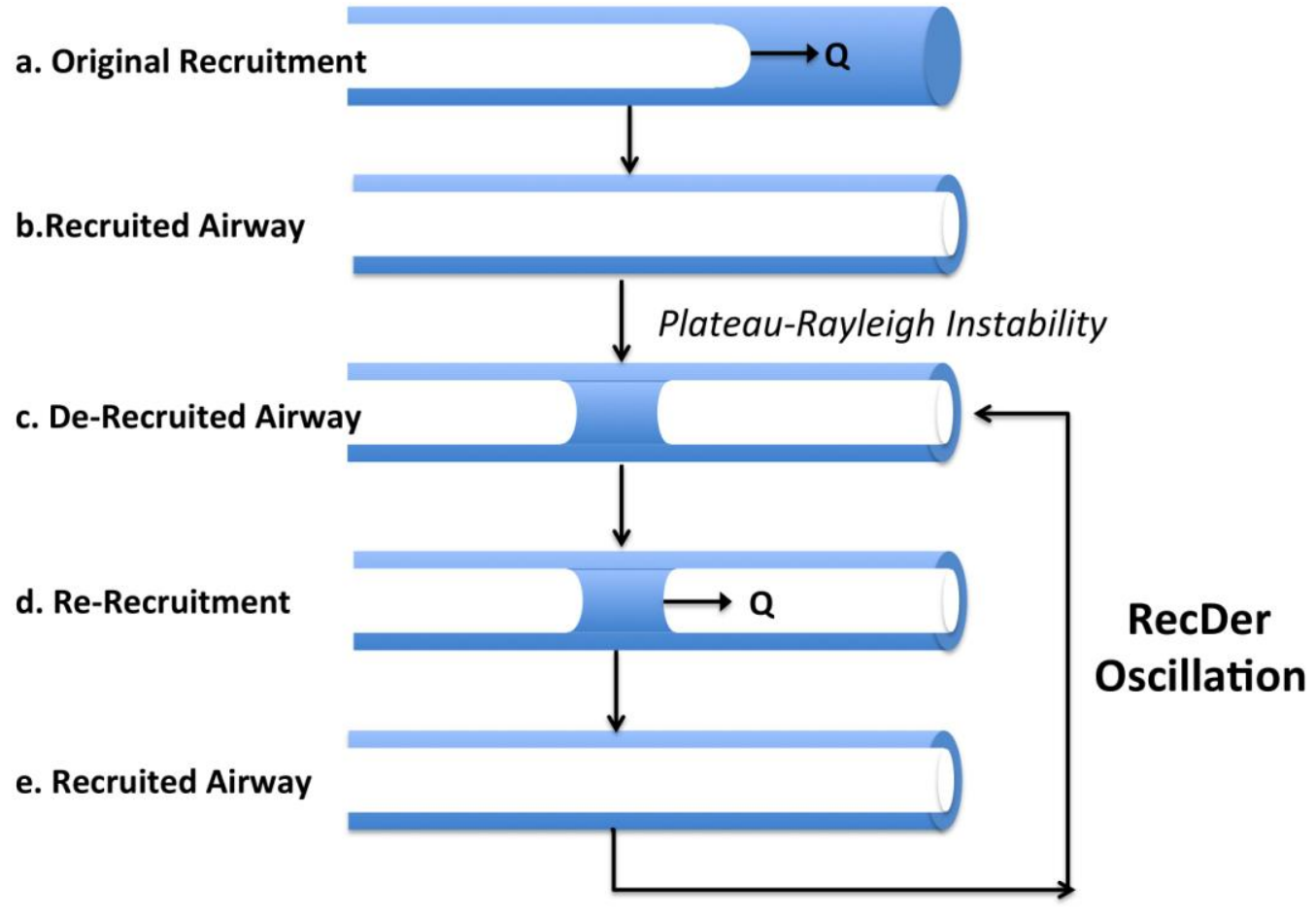

Figure 1: Schematic of the Recruitment/De-recruitment (RecDer) dynamics that are investigated in this model. Note that although not illustrated, the tube is compliant and may undergo cross-sectional buckling as a result of a negative transmural pressure. 


\section{Tube Law}

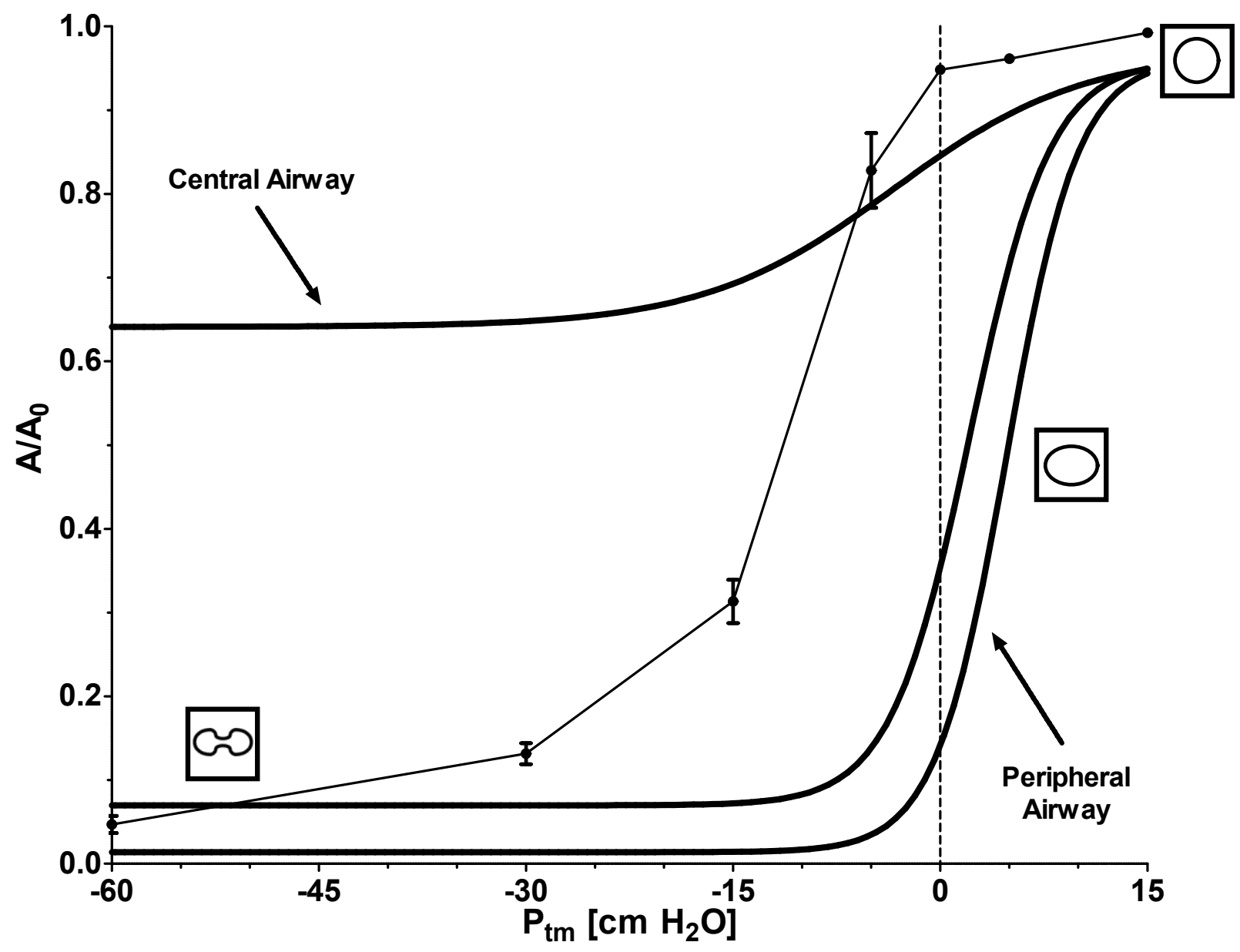

Figure 2: Representative compliance representation (tube-law) representing a change in cross-sectional area $\left(A / A_{0}\right)$ as a function of transmural pressure, $P_{t m}$. Bold lines represent pulmonary airways, and data and narrow line represents the PDMS tube used in this study. 


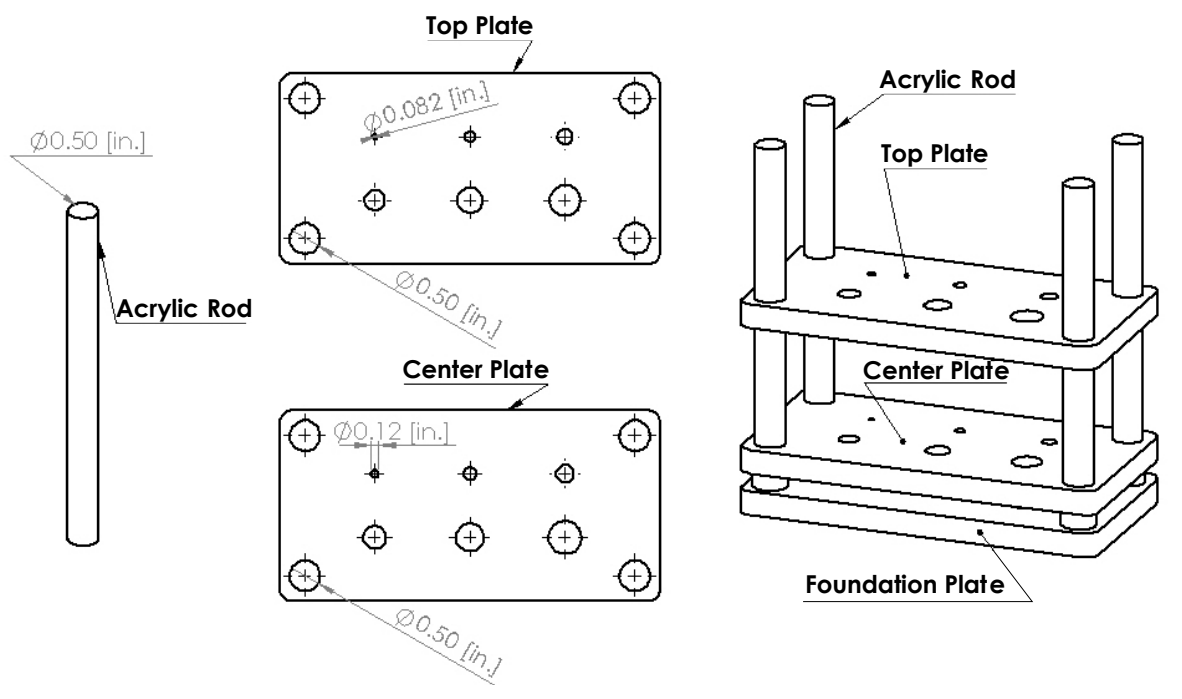

Figure 3: Device that creates uniform thickness in the PDMS tubes. Not to scale. 


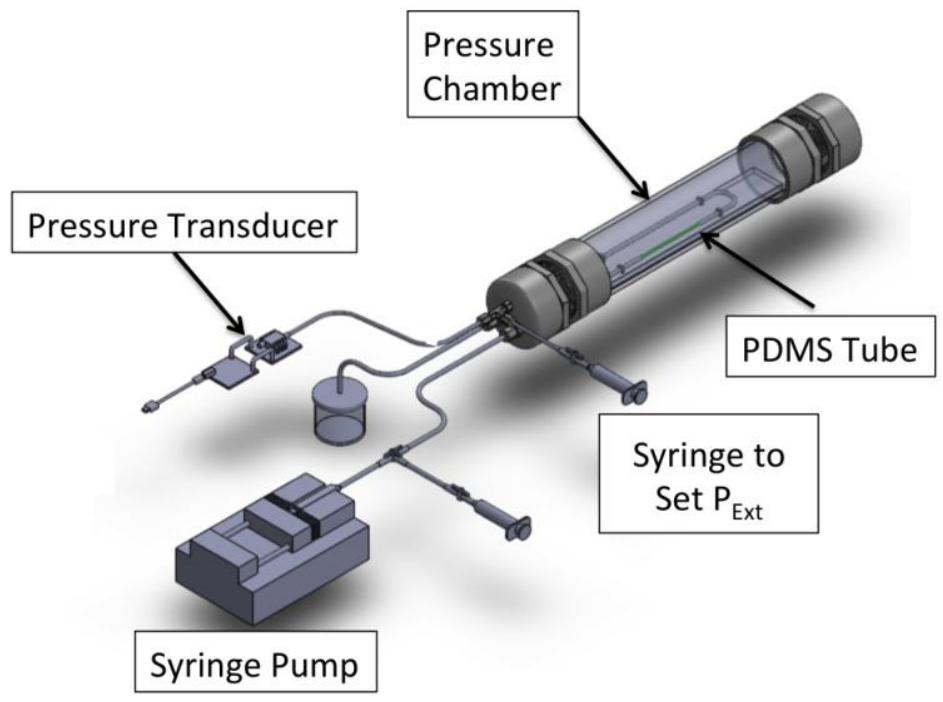

Figure 4: Rendering of the entire pressure chamber. Not to scale. 


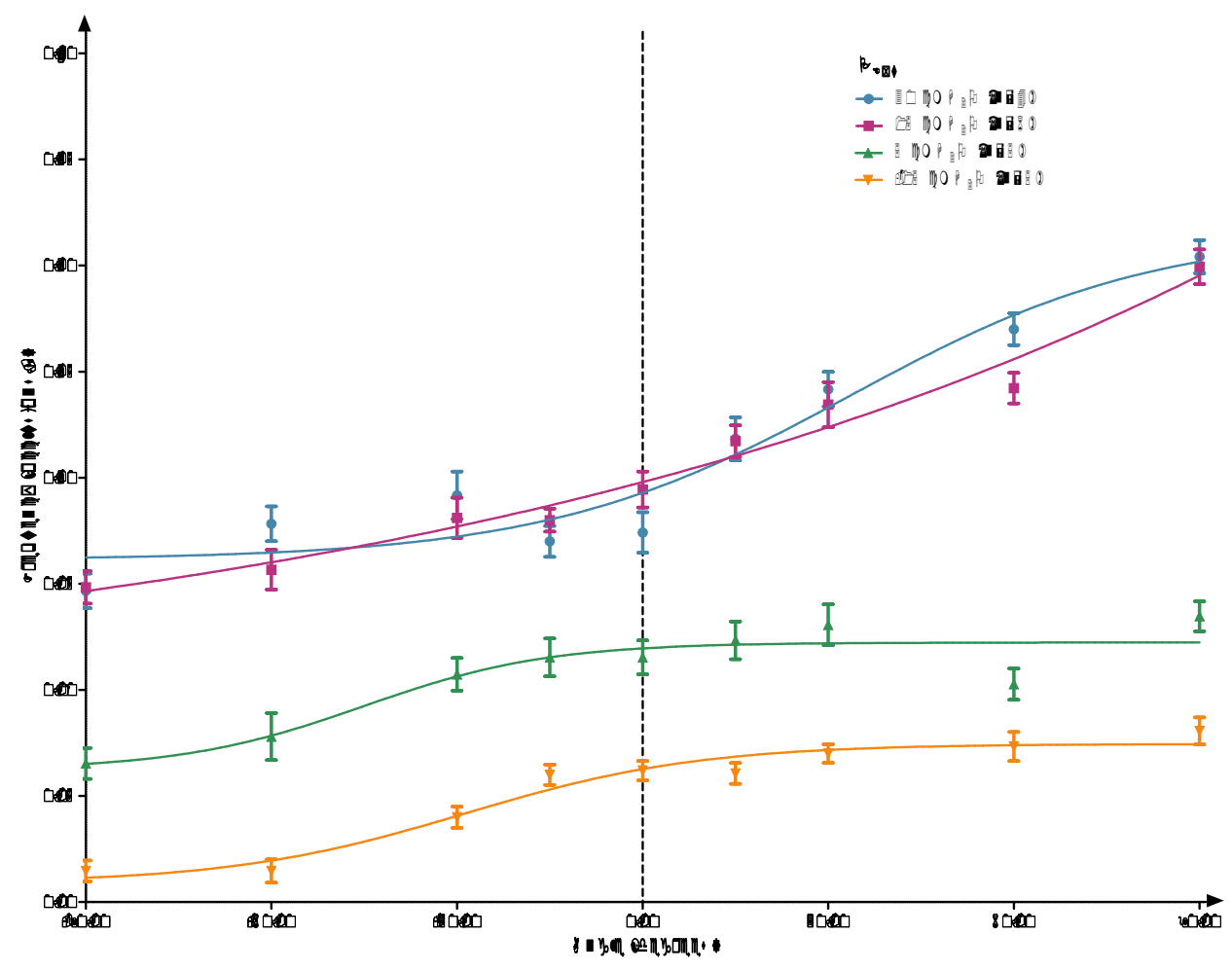

Figure 5: Average interfacial instability frequency vs. for $P_{E x t}=30,15,5$ and $-15 \mathrm{cmH}_{2} 0$. 


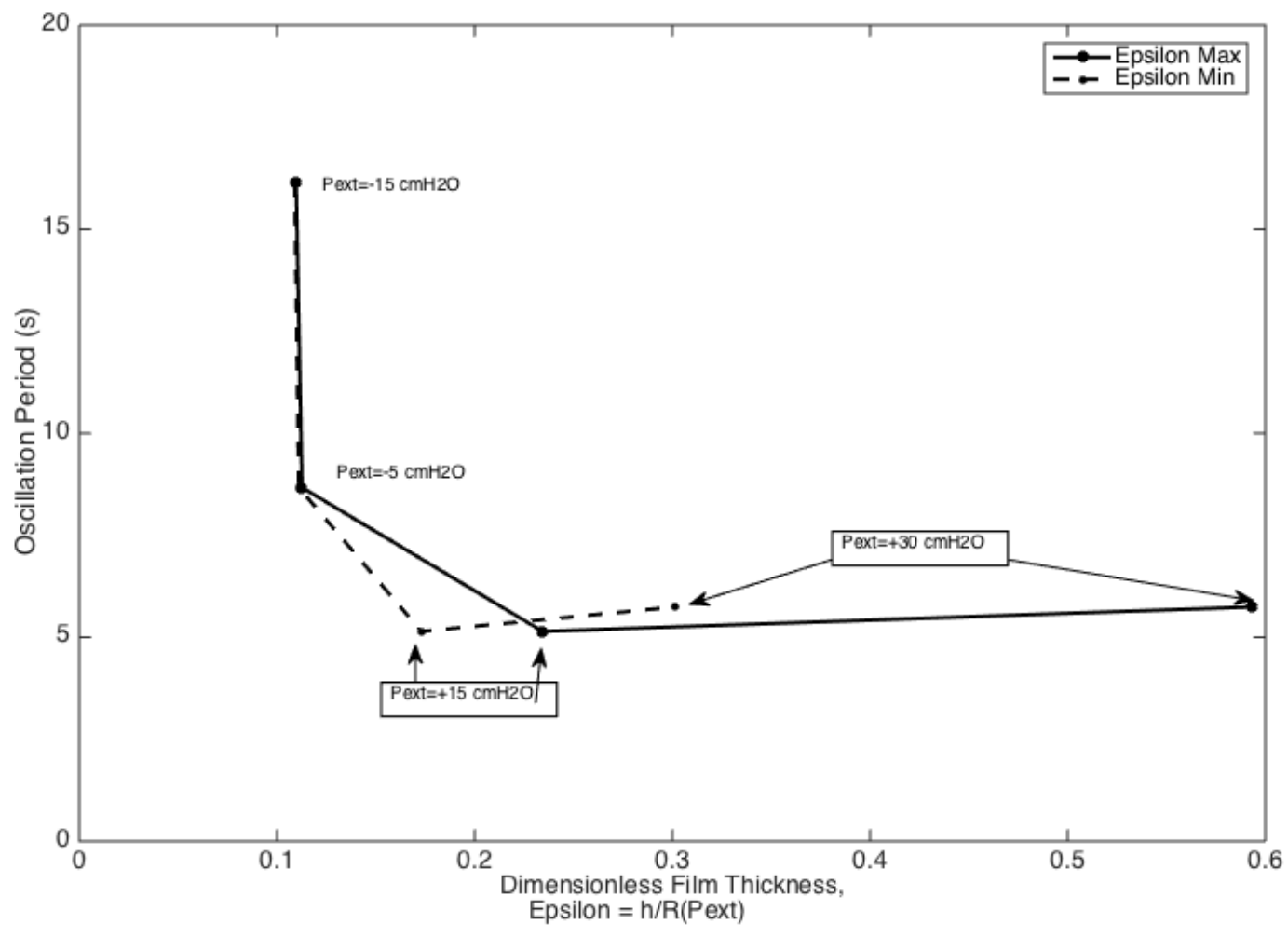

Figure 6: Relationship between the oscillation period and the estimated dimensionless film thickness. 

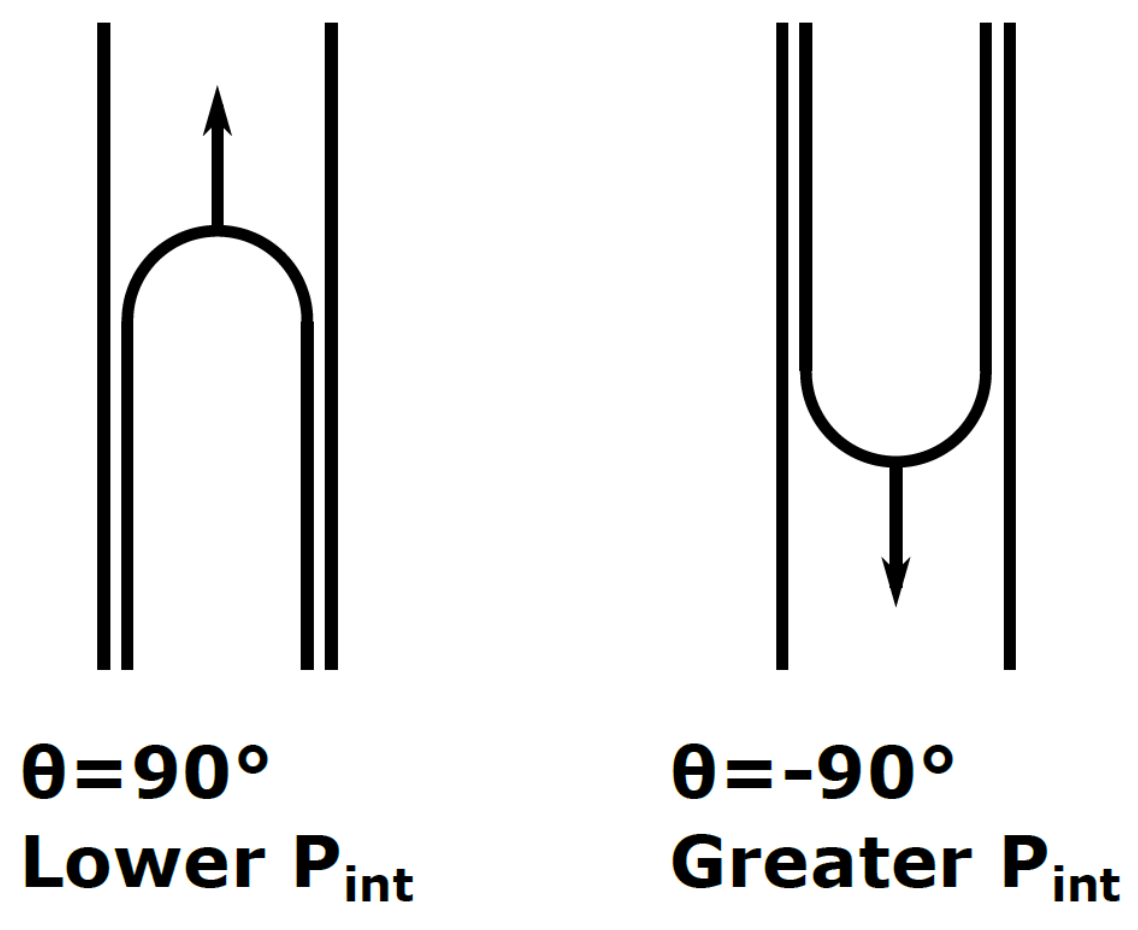

Figure 7: Schematic of a finger of air in a compliant tube model at angles of inclination $\theta= \pm 90^{\circ}$. An increase in $P$ Int is hypothesized to improve the stability and reduce RecDer oscillation. 